\begin{tabular}{|c|c|}
\hline Title & $\begin{array}{l}\text { Theoretical Prediction of Thermooptical and Structurally Disordered Sensitivities in Metallo-Dielectric Photonic } \\
\text { Crystals }\end{array}$ \\
\hline Author(s) & Florous, Nikolaos John; Saitoh, Kunimasa; Koshiba, Masanori \\
\hline Citation & $\begin{array}{l}\text { IEEE Photonics Technology Letters, 18(7), } 898-900 \\
\text { https://doi.org/10.1109/LPT.2006.872286 }\end{array}$ \\
\hline Issue Date & 2006-04-01 \\
\hline Doc URL & http:/hdl .handle.net/2115/8510 \\
\hline Rights & $\begin{array}{l}\text { (c) } 2006 \text { IEEE. Personal use of this material is permitted. However, permission to reprint/republish this material for } \\
\text { advertising or promotional purposes or for creating new collective works for resale or redistribution to servers or lists, } \\
\text { or to reuse any copyrighted component of this work in other works must be obtained from the IEEE. }\end{array}$ \\
\hline Type & article \\
\hline File Information & 01608199.pdf \\
\hline
\end{tabular}

Instructions for use 


\title{
Theoretical Prediction of Thermooptical and Structurally Disordered Sensitivities in Metallo-Dielectric Photonic Crystals
}

\author{
Nikolaos John Florous, Member, IEEE, Kunimasa Saitoh, Member, IEEE, and Masanori Koshiba, Fellow, IEEE
}

\begin{abstract}
In this letter, we introduce a novel computational technique for predicting thermal and structural sensitivities in metallo-dielectric photonic crystals (PCs). The computational scheme is based on a hybrid formalism of the scattering matrix technique combined with the adjoint network method. The proposed technique can predict with high accuracy the impact of temperature fluctuations or structural disorders, to the lightwave propagation in PCs, without additional computational effort. Numerical simulations show that PC circuits based on metallic-metamaterial platforms are significantly less sensitive to temperature variations in comparison to usual dielectric PCs.
\end{abstract}

Index Terms-Lightwave propagation, metamaterials, photonic crystal (PC) circuits, sensitivity.

\section{INTRODUCTION}

$\mathbf{P}$ HOTONIC crystals (PCs) have recently attracted considerable attention by optical scientists, because they can radically alter the optical properties of matter in an extraordinary way [1].

In practical realization of PC devices, it is of fundamental importance to accurately predict the sensitivity of the device properties to fabrication tolerances. Sensitivity analysis of environmental temperature fluctuations is also important, especially for characterizing the thermal stability of PC devices, and can be particularly helpful in deciding whether to use a special temperature maintaining environment to stabilize transmission. Until recently, however, such sensitivity studies have been reported only for structural disorders, and in particular for PCs composed of usual dielectric materials [2]-[5].

The inclusion of metals in PCs, operating at optical wavelengths, has attracted significant attention due to their surprising ultralow refractive index metamaterial characteristics [6], [7]. However, the sensitivity properties of metallic PC metamaterials have not been reported so far, mainly for two reasons. First, a general technique which permits the calculation of both structural and temperature sensitivities was not known. Second, for the optical characterization of metallic materials, a simplified temperature-independent Drude model has been used so far [8], thus prohibiting the thermal properties of these nanocomposites to be studied. Taking all the above circumstances into account, in this letter, we describe a novel sensitivity analysis technique based on a hybrid formalism of the scattering matrix method

Manuscript received August 26, 2005; revised January 11, 2006.

The authors are with the Division of Media and Network Technologies, Hokkaido University, Sapporo 060-0814, Japan (e-mail: nflorous@dpo7.ice.eng.hokudai.ac.jp; koshiba@ist.hokudai.ac.jp).

Digital Object Identifier 10.1109/LPT.2006.872286
[9]-[11] with the adjoint network method [12], [13], for performing fast and accurate sensitivity calculations in PCs. Such an analytical technique which permits the study of both structural and temperature fluctuations in PCs will be reported for the fist time. In particular, we show through numerical simulations that the use of metallic metamaterial PCs significantly reduces the sensitivity to temperature fluctuations in comparison to usual dielectric PCs.

\section{HYbrid COMPUTATIONAL SCHEME FOR CALCULATING SENSITIVITIES IN PCs}

In the standard analysis of PC circuits using the scattering matrix technique [9]-[11], we arrive at a matrix equation for the unknown expansion coefficients for the field distribution $B_{j}, j=1,2, \ldots, N$, where $N$ is the total number of cylinders in the PC under consideration, which has the following form:

$$
\begin{array}{r}
{\left[\begin{array}{cccc}
I & -S_{1} T_{1,2} & \cdots & -S_{1} T_{1, N} \\
-S_{2} T_{2,1} & I & \cdots & -S_{2} T_{2, N} \\
\vdots & \vdots & \ddots & \vdots \\
-S_{N} T_{N, 1} & -S_{N} T_{N, 2} & \cdots & I
\end{array}\right]\left[\begin{array}{c}
B_{1} \\
B_{2} \\
\vdots \\
B_{N}
\end{array}\right]} \\
=\left[\begin{array}{c}
S_{1} Q_{1} \\
S_{2} Q_{2} \\
\vdots \\
S_{N} Q_{N}
\end{array}\right]
\end{array}
$$

where $I$ denotes the unit matrix, and $S_{j}, T_{j, k}, Q_{j}$ are quantities defined explicitly in terms of Hankel and Bessel functions for $j=1,2, \ldots, N$, and $k=-N, \ldots,+N$ and can be found in [9] and [11]. Using standard notations from the method of moments, we can rewrite (1) in the following compact form:

$$
\overline{\bar{Z}}(\mathbf{x}) \cdot \bar{B}=\bar{V} .
$$

Here, $x$ is the vector of design parameters, $\bar{B}$ is the state vector with the unknown coefficients of the field distribution, $\bar{V}$ is the global excitation vector which carries the information of the source excitation and the Sommerfeld radiation condition at infinity, and $\bar{Z}(\mathbf{x})$ is considered to be a nonsingular interaction matrix which carries all the information for the structural and material properties of the PC under consideration. Thus, we restrict our analysis in PCs with low coupling between the waveguide channel and coherent cladding modes. A global objective function which can be any electromagnetic quantity (e.g., transmission characteristics in which we want to calculate the sensitivity response) that is differentiation with respect to the design parameter- $\boldsymbol{x}$, is defined explicitly as $f(\mathbf{x}, \bar{B}(\mathbf{x}))$. From 


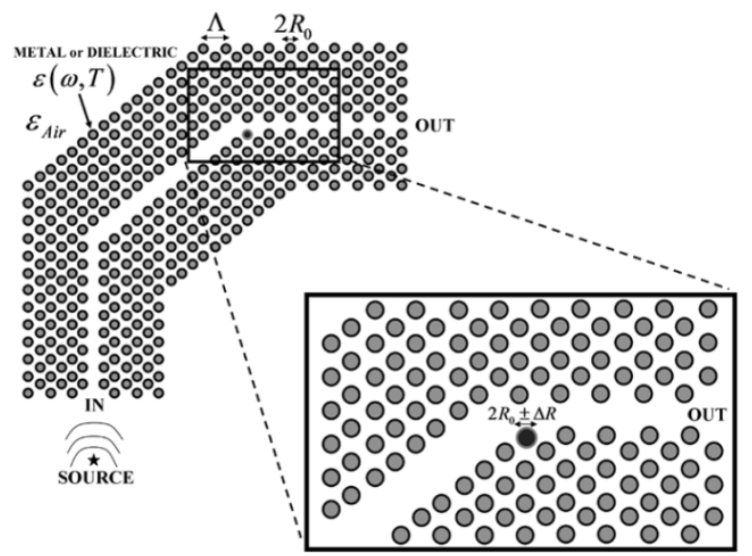

Fig. 1. PC multiwaveguide bend. Magnified picture shows details around the second corner with a single structural defect.

the mathematical point of view, the objective is to perform the following differentiation subjected to a matrix constrain:

$$
\nabla_{\mathbf{x}} f, \quad \text { subjected to } \overline{\bar{Z}}(\mathbf{x}) \cdot \bar{B}=\bar{V}
$$

where $\nabla_{\mathbf{x}}=\left[\partial / \partial x_{1}, \partial / \partial x_{2}, \ldots, \partial / \partial x_{n}\right]$ and $n$ stands for the total number of design parameters. From (1), with direct differentiation, we obtain

$$
\nabla_{\mathbf{x}} \bar{B}=\overline{\bar{Z}}^{-1}\left[\nabla_{\mathbf{x}} \bar{V}-\nabla_{\mathbf{x}}(\overline{\bar{Z}} \cdot \bar{B})\right] .
$$

Although (4) can be subsequently used for calculating exact sensitivities, we are mainly interested in the computation of the sensitivity of the global objective function $\nabla_{\mathbf{x}} f$ rather than the sensitivity of the state variable $\nabla_{\mathbf{x}} \bar{B}$. In such a case, the use of the adjoint network method [13] provides an efficient computational scheme which can be summarized in the following expressions:

$$
\nabla_{\mathbf{x}} f=\nabla_{\mathbf{x}}^{e} f+\hat{B}^{T}\left[\nabla_{\mathbf{x}} \bar{V}-\nabla_{\mathbf{x}}(\overline{\bar{Z}} \cdot \bar{B})\right]
$$

where $\nabla_{\mathbf{x}}^{e} f$ reflects the explicit behavior of the global objective function on the vector of design parameters $\mathbf{x}$ and on the state vector $\bar{B}$. The adjoint variable vector $\hat{B}$ is a solution to the following matrix equation:

$$
\overline{\bar{Z}}^{T} \cdot \hat{B}=\left[\nabla_{\bar{B}} f\right]^{T}
$$

Equation (5) combined with (6) form the basis for the adjoint network method. In its present form, the computational scheme entirely depends on analytical expressions of the matrices under consideration and, thus, significantly defers from other adjoint computational schemes used so far. From this viewpoint, the proposed scheme is novel, and will be applied for the first time to characterize thermal sensitivities in PCs.

\section{NUMERICAL RESULTS AND DISCUSSION}

To show the applicability and the usefulness of this technique, we consider as a test circuit the PC architecture shown in Fig. 1. It is composed of dielectric or metallic nanowires (pillars), arranged in a hexagonal configuration with lattice constant $\Lambda=500 \mathrm{~nm}$ and radii $R_{0}=100 \mathrm{~nm}$. To accurately model the material characteristics of metallic nano-pillars, we have adopted a temperature-dependent Drude model [14]

$$
\begin{aligned}
\varepsilon_{\text {metal }} & =1-\varepsilon_{\infty} \frac{\omega_{p}^{2}+v_{c}^{2}}{\omega^{2}+v_{c}^{2}}+j \varepsilon_{\infty} \frac{\omega_{p}^{2}+v_{c}^{2}}{\omega} \frac{v_{c}}{\omega^{2}+v_{c}^{2}}, v_{c}(\omega, T) \\
& =v_{c 0}\left[1+\left(\frac{h \omega}{2 \pi k_{B} T}\right)^{2}\right]
\end{aligned}
$$

where $\varepsilon_{\infty}$ is the relative dielectric constant at high frequencies, $\omega_{p} /(2 \pi)=2175 \mathrm{THz}$ is the plasma frequency, $v_{c 0} /(2 \pi)=$ $4.35 \mathrm{THz}$ is the classical relaxation rate in the free electron model of metals, $\omega$ is the angular frequency, $T$ is the absolute temperature, $k_{B}$ is the Boltzman's constant, and $h$ is the Plank's constant. The above parameters correspond to silver $(\mathrm{Ag})$ which will be considered as a model metal. In the case of usual dielectric materials, we consider the following Drude-Lorentz type of dielectric permittivity [15] of intrinsic semiconductors

$$
\begin{aligned}
\varepsilon(\omega, T) & =\varepsilon_{d}\left[1-\frac{\omega_{p}^{2}(T)}{\omega^{2}}\right] \\
\omega_{p}^{2}(T) & =\sum_{i} \frac{4 \pi n_{i} e^{2}}{m_{i} \varepsilon_{d}}, n_{e}(T) \\
& =5.76 \times 10^{14} T^{3 / 2} \exp \left(-\frac{0.13}{k_{B} T}\right)
\end{aligned}
$$

where $\varepsilon_{d}=17.7, m_{e} / m=0.015, n_{i}$ is the density of the free electrons and holes in the relevant conduction $(i=e)$ and valance band $(i=v)$, and $m_{i} / m$ stands for their effective masses. These parameters correspond to the intrinsic semiconductor known as InSb. In our investigations, however, we will also consider the case of GaAs, which has larger electronic band-gap. Additionally, we assume that the geometrical characteristics of the pillars will remain invariant as the temperature changes and only the permittivity function is affected.

\section{A. Validation of the Proposed Computational Scheme}

In order to validate our computer code, we perform a comparison with already reported calculations of structural sensitivities in usual semiconducting PCs (GaAs rods in air). Following the example of [2, Fig. 13], Fig. 2 shows the results obtained with our method (solid line) and the finite-element method used in [2] (dotted line). We can clearly see that there is a good agreement between the two methods, a fact that ensures the accuracy and the efficiency of our computer code.

\section{B. Computation of Structural and Thermal Sensitivities}

To demonstrate the calculation of structural sensitivities (first-order derivative of the global objective function with respect to the design parameter) of the device in Fig. 1, we consider as a global objective function the transmission characteristics $\operatorname{Tr}(\omega, \Delta R)=P_{\mathrm{OUT}} / P_{\mathrm{IN}}$, at constant temperature $T_{0}=300 \mathrm{~K}$, and as a vector of design parameter the single scalar variable $\Delta R$, which is the perturbed portion of the radius of the single defect, as shown in Fig. 1. The upper panel in Fig. 3 shows the sensitivity of the transmission curve as a function of the normalized perturbation $\Delta R / R_{0}$, for dielectric-InSb (dotted line), or metallic-Ag nanopillars (solid line). From these results, we can conclude that the dynamic rate of change 


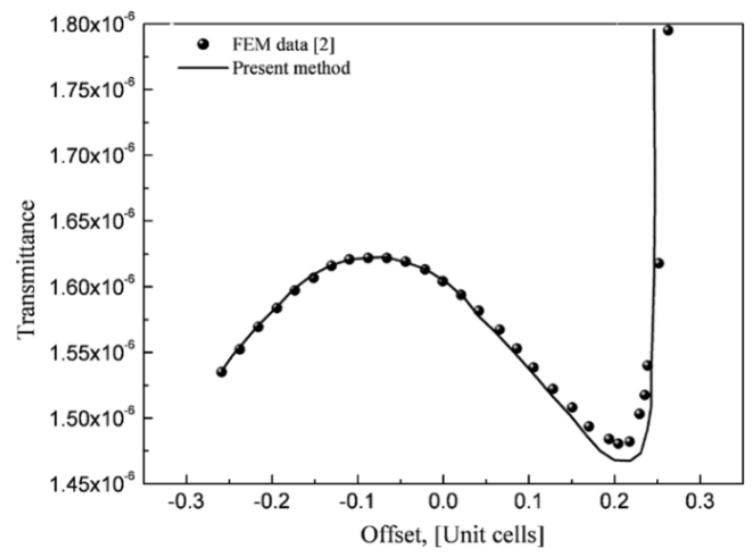

Fig. 2. Validation results between our computer code (solid line) and finite-element method used in [2] (dotted line), for the impact of a line-defect offset in the transmittivity of a PC waveguide, as a function of the offset (unit cells). We can see a very good agreement between the two methods.
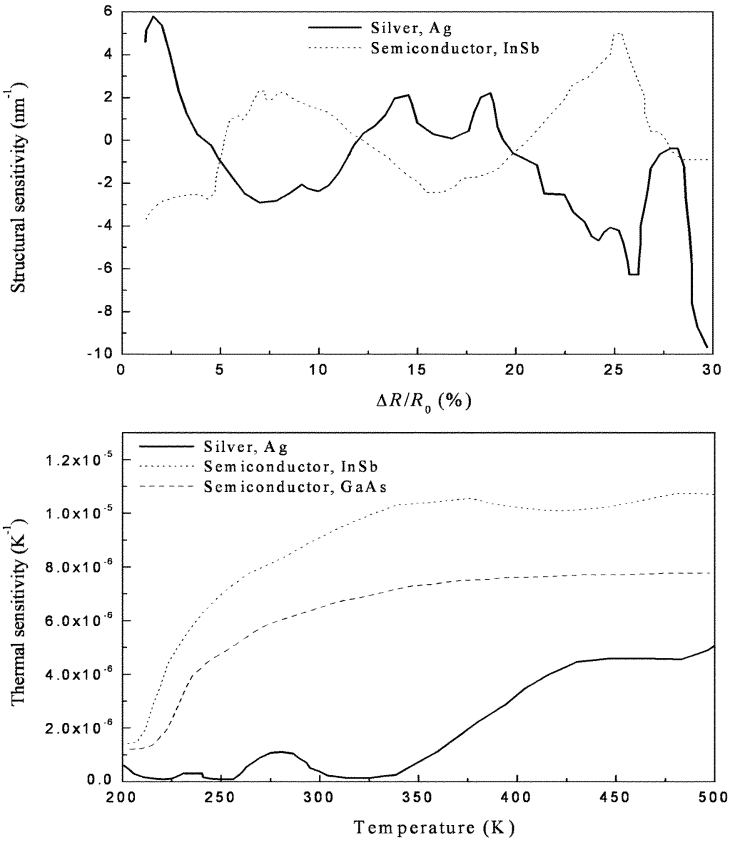

Fig. 3. Upper panel: structural sensitivity of the transmission as a function of the defect tolerance $\Delta R / R_{0}$, for silver-Ag (solid line) and dielectric-InSb (dotted line). Lower panel: absolute thermal sensitivity as a function of the temperature, for silver-Ag (solid line), InSb (dotted line), and GaAs (dashed line).

in the transmission curve, namely the difference between local minima and maxima of the sensitivity curve is greater in case of the metallic-defect, than that of the dielectric, especially for higher tolerances $(13 \%-30 \%)$. The physical explanation for this result is that the metallic nanowires interact more strongly with light, in the visible and near-infrared frequency bands, and as a result they are found to have more pronounced reflectivity. As a consequence, a high structural variation of the metallic nanowires, especially in the case where they are positioned near discontinuities of waveguide bends, will result as a general rule in larger sensitivities than the case of dielectric defected nanowires. The lower panel in Fig. 3 demonstrates the calculation of thermal sensitivities (first-order derivative of the global objective function with respect to the temperature), at a frequency- $\omega_{0}$ corresponding to maximum transmittivity, in case of $\Delta R=0$. The calculation is performed for silver (Ag), and for semiconducting materials, InSb (dotted line) and GaAs (dashed line). From these results, we can clearly see that the metallic-based platform is less sensitive to thermal fluctuations. In addition, we conclude that semiconductors with a larger electronic bandgap (GaAs) exhibit lower sensitivity in comparison to semiconductors with a smaller electronic bandgap (InSb). Even in such cases, the sensitivity of the metallic platform is at least two to five times smaller than that of GaAs, over a wide range of temperatures.

\section{CONCLUSION}

An efficient analytical computational scheme for fast and accurate predictions of thermal and structural sensitivities in metallo-dielectric PCs has been proposed. We have demonstrated that metallic-based metamaterial PCs are less sensitive to temperature variations than usual PCs based on dielectric platforms. A generalized stochastic method for yield analysis in PCs with random structural disorders is currently under consideration.

\section{REFERENCES}

[1] J. D. Joannopoulos, R. D. Meade, and J. N. Winn, Photonic Crystals: Molding the Flow of Light. Princenton, NJ: Princeton Univ. Press, Sep. 1995.

[2] W. R. Frey and H. T. Johnson, "Finite element analysis of disorder effects in photonic crystals," Phys. Rev. B, vol. 70, p. 165 116, Oct. 2004.

[3] A. Chutinan and S. Noda, "Effects of structural fluctuations on the photonic bandgap during fabrication of a photonic crystal," J. Opt. Soc. Amer. B, vol. 16, pp. 240-244, Feb. 1999.

[4] A. A. Asatryan, P. A. Robinson, L. C. Botten, R. C. McPhedran, N. A. Nicorovici, and C. M. Stekre, "Effects of disorder on wave propagation in two-dimensional photonic crystals," Phys. Rev. E, vol. 60, pp. 6118-6127, Nov. 1999.

[5] Z.-Y. Li and Z.-Q. Zhang, "Fragility of photonic band gaps in inverseopal photonic crystals," Phys. Rev. B, vol. 62, pp. 1516-1519, Jul. 2000.

[6] B. T. Schwartz and R. Piestun, "Total external reflection from metamaterials with ultralow refractive index," J. Opt. Soc. Amer. B, vol. 20, pp. 2448-2453, 2003

[7] V. Poborchii, T. Taya, T. Kanayama, and A. Moroz, "Silver-coated silicon pillar photonic crystals: Enhancement of a photonic band gap," Appl. Phys. Lett., vol. 82, pp. 508-510, 2003.

[8] V. Kuzmiak, A. A. Maradudin, and F. Pincemin, "Photonic band structures of two-dimensional systems containing metallic components," Phys. Rev. B, vol. 50, pp. 16843-16844, Dec. 1994.

[9] D. Felbacq, G. Tayeb, and D. Maystre, "Scattering by a random set of parallel cylinders," J. Opt. Soc. Amer. A, vol. 11, pp. 2526-2538, Sep. 1994.

[10] R. C. McPhedran, L. C. Botten, A. A. Asatryan, N. A. Nicorovici, P. A. Robinson, and C. M. Stekre, "Calculation of electromagnetic properties of regular and random arrays of metallic and dielectric cylinders," Phys. Rev. E, vol. 60, pp. 7614-7618, Dec. 1999.

[11] N. J. Florous and M. Koshiba, "Theoretical prediction of light-wave localization mechanisms in metallo-dielectric photonic crystal circuits," in Integrated Photonic Research and Applications (IPR 2005), San Diego, CA, Apr. 2005

[12] F. Alessandri, M. Mongiardo, and R. Sorrentino, "New efficient full wave optimization of microwave circuits by the adjoint network method," IEEE Microw. Guided Wave Lett., vol. 3, no. 11, pp. 414-416, Nov. 1993.

[13] J. W. Bandler and R. E. Seviora, "Wave sensitivities of networks," IEEE Trans. Microw. Theory Tech., vol. MTT-20, no. 2, pp. 138-147, Feb. 1972.

[14] F. Abeles, Optical Properties of Solids. Amsterdam, The Netherlands: North-Holland, 1972.

[15] P. Halevi and E. R. Mendieta, "Tunable photonic crystals with semiconducting constituents," Phys. Rev. Lett., vol. 85, pp. 1875-1878, Aug. 2000. 